\title{
STRONG SUBDIFFERENTIABILITY AND LOCAL BISHOP-PHELPS-BOLLOBÁS PROPERTIES
}

\author{
SHELDON DANTAS, SUN KWANG KIM, HAN JU LEE, AND MARTIN MAZZITELLI
}

\begin{abstract}
It has been recently presented in [21] some local versions of the Bishop-Phelps-Bollobás type property for operators. In the present article, we continue studying these properties for multilinear mappings. We show some differences between the local and uniform versions of the Bishop-PhelpsBollobás type results for multilinear mappings, and also provide some interesting examples which shows that this study is not just a mere generalization of the linear case. We study those properties for bilinear forms on $\ell_{p} \times \ell_{q}$ using the strong subdifferentiability of the norm of the Banach space $\ell_{p} \hat{\otimes}_{\pi} \ell_{q}$. Moreover, we present necessary and sufficient conditions for the norm of a Banach space $Y$ to be strongly subdifferentiable through the study of these properties for bilinear mappings on $\ell_{1}^{N} \times Y$.
\end{abstract}

\section{INTRODUCTION}

In Banach space theory, it is well-known that the set of all norm attaining continuous linear functionals defined on a Banach space $X$ is dense in its topological dual space $X^{*}$. This is the famous Bishop-Phelps theorem [9]. In 1970, this result was strengthened by Bollobás, who proved a quantitative version in the following sense: if a norm-one linear functional $x^{*}$ almost attains its norm at some $x$, then, near to $x^{*}$ and $x$, there are, respectively, a new norm-one functional $y^{*}$ and a new point $y$ such that $y^{*}$ attains its norm at $y$ (see [10, Theorem 1]). Nowadays, this result is known as the Bishop-Phelps-Bollobás theorem and it has been used as an important tool in the study of Banach spaces and operators. For example, it was used to prove that the numerical radius of a continuous linear operator is the same as its adjoint.

It is natural to ask whether the Bishop-Phelps and Bishop-Phelps-Bollobás theorems hold also for bounded linear operators. In 1963, Lindenstrauss gave the first example of a Banach space $X$ such that the set of all norm attaining operators on $X$ is not dense in the set of all bounded linear operators (see [31, Proposition 5]). On the other hand, he studied some conditions on the involved Banach spaces in order to get a Bishop-Phelps type theorem for operators. For instance, he proved that the set of all operators whose second adjoint attain their norms is dense, so, in particular, if $X$ is a reflexive Banach space, then the set of all norm attaining operators is dense for arbitrary range spaces (actually, this result was extended by Bourgain in [12, Theorem 7] by showing that the Radon-Nikodým property implies the same result). This topic has been considered by many authors and we refer the reader to the survey paper [1] for more information and background about denseness of norm attaining operators. On the other hand, M. Acosta, R. Aron, D. García, and M. Maestre studied the vector-valued case of the Bishop-Phelps-Bollobas theorem and introduced [3] the Bishop-Phelps-Bollobás property.

Now we introduce the notation and necessary preliminaries. Let $N$ be a natural number. We use capital letters $X, X_{1}, \ldots, X_{N}, Y$ for Banach spaces over a scalar field $\mathbb{K}$ which can be the field of the real numbers $\mathbb{R}$ or the field of the complex numbers $\mathbb{C}$. The closed unit ball and the unit sphere of

2010 Mathematics Subject Classification. Primary 46B04; Secondary 46B07, 46B20.

Key words and phrases. Banach space; norm attaining operators; Bishop-Phelps-Bollobás property.

The first author was supported by the project OPVVV CAAS CZ.02.1.01/0.0/0.0/16_019/0000778. The second author was partially supported by Basic Science Research Program through the National Research Foundation of Korea(NRF) funded by the Ministry of Education, Science and Technology (NRF-2017R1C1B1002928). The third author was supported by the Research program through the National Research Foundation of Korea funded by the Ministry of Education, Science and Technology (NRF-2016R1D1A1B03934771). The fourth author was partially supported by CONICET PIP 11220130100329 CO. 
$X$ are denoted by $B_{X}$ and $S_{X}$, respectively. The topological dual space of $X$ is denoted by $X^{*}$ and $\mathcal{L}\left(X_{1}, \ldots, X_{N} ; Y\right)$ stands for the set of all bounded $N$-linear mappings from $X_{1} \times \cdots \times X_{N}$ into $Y$. For the convenience, if $X_{1}=\ldots=X_{N}=X$, then we use the shortened notation $\mathcal{L}\left({ }^{N} X ; Y\right)$. When $N=1$, we have the set of all bounded linear operators from $X$ into $Y$, which we denote simply by $\mathcal{L}(X ; Y)$.

We say that an $N$-linear mapping $A \in \mathcal{L}\left(X_{1}, \ldots, X_{N} ; Y\right)$ attains its norm if there exists $\left(z_{1}, \ldots, z_{N}\right) \in$ $S_{X_{1}} \times \cdots \times S_{X_{N}}$ such that $\left\|A\left(z_{1}, \ldots, z_{N}\right)\right\|=\|A\|$, where $\|A\|=\sup \left\|A\left(x_{1}, \ldots, x_{N}\right)\right\|$, the supremum being taken over all the elements $\left(x_{1}, \ldots, x_{N}\right) \in S_{X_{1}} \times \cdots \times S_{X_{N}}$. We denote by $\mathrm{NA}\left(X_{1}, \ldots, X_{N} ; Y\right)$ the set of all norm attaining $N$-linear mappings.

Definition $1.1([4,14,17,30])$. We say that $\left(X_{1}, \ldots, X_{N} ; Y\right)$ has the Bishop-Phelps-Bollobás property for $N$-linear mappings (BPBp for $N$-linear mappings, for short) if given $\varepsilon>0$, there exists $\eta(\varepsilon)>0$ such that whenever $A \in \mathcal{L}\left(X_{1}, \ldots, X_{N} ; Y\right)$ with $\|A\|=1$ and $\left(x_{1}, \ldots, x_{N}\right) \in S_{X_{1}} \times \cdots \times S_{X_{N}}$ satisfy

$$
\left\|A\left(x_{1}, \ldots, x_{N}\right)\right\|>1-\eta(\varepsilon),
$$

there are $B \in \mathcal{L}\left(X_{1}, \ldots, X_{N} ; Y\right)$ with $\|B\|=1$ and $\left(z_{1}, \ldots, z_{N}\right) \in S_{X_{1}} \times \cdots \times S_{X_{N}}$ such that

$$
\left\|B\left(z_{1}, \ldots, z_{N}\right)\right\|=1, \max _{1 \leqslant j \leqslant N}\left\|z_{j}-x_{j}\right\|<\varepsilon, \quad \text { and }\|B-A\|<\varepsilon .
$$

When $N=1$, we simply say that the pair $(X ; Y)$ satisfies the BPBp (see [3, Definition 1.1]). Note that the Bishop-Phelps-Bollobás theorem asserts that the pair $(X ; \mathbb{K})$ has the BPBp for every Banach space $X$. It is immediate to notice that if the pair $(X ; Y)$ has $\mathbf{B P B}$, then $\overline{\mathrm{NA}(X ; Y)}=\mathcal{L}(X ; Y)$. However, the converse is not true even for finite dimensional spaces. Indeed, for a finite dimensional Banach space $X$, the fact that $B_{X}$ is compact implies that every bounded linear operator on $X$ attains its norm, but it is known that there is some Banach space $Y_{0}$ so that the pair $\left(\ell_{1}^{2} ; Y_{0}\right)$ fails the $\mathbf{B P B}$ (see $[7$, Example 4.1]). This shows that the study of the BPBp is not just a trivial extension of that of the density of norm attaining operators.

Similar to the case of operators, there were a lot of attention to the study of the denseness of norm attaining bilinear mappings. It was proved that, in general, there is no Bishop-Phelps theorem for bilinear mappings (see [2, Corollary 4]). Moreover, it is known that $\overline{\mathrm{NA}\left({ }^{2} L^{1}[0,1] ; \mathbb{K}\right)} \neq \mathcal{L}\left({ }^{2} L^{1}[0,1] ; \mathbb{K}\right)$ (see [13, Theorem 3]), even though $\overline{\mathrm{NA}\left(L^{1}[0,1] ; L_{\infty}[0,1]\right)}=\mathcal{L}\left(L^{1}[0,1] ; L_{\infty}[0,1]\right)$ (see [24]). This result is interesting since the Banach space $\mathcal{L}\left(X_{1}, X_{2} ; \mathbb{K}\right)$ is isometrically isomorphic to $\mathcal{L}\left(X_{1} ; X_{2}^{*}\right)$ via the canonical isometry $A \in \mathcal{L}\left(X_{1}, X_{2} ; \mathbb{K}\right) \longmapsto T_{A} \in \mathcal{L}\left(X_{1} ; X_{2}^{*}\right)$ given by $\left[T_{A}\left(x_{1}\right)\right]\left(x_{2}\right)=A\left(x_{1}, x_{2}\right)$. Concerning the BPBp for bilinear mappings, it is known that $\left(\ell_{1}, \ell_{1} ; \mathbb{K}\right)$ fails the BPBp for bilinear mappings (see [14]) but the pair $\left(\ell_{1}, Y\right)$ satisfies the BPBp for many Banach spaces $Y$, including $\ell_{\infty}$ (see $[3$, Section 4$]$ ). We refer the papers $[4,17,30]$ for more results on the $\mathbf{B P B p}$ for multilinear mappings.

Very recently, a stronger property than the BPBp was defined and studied.

Definition $1.2([18,19])$. We say that $\left(X_{1}, \ldots, X_{N} ; Y\right)$ has the Bishop-Phelps-Bollobás point property for $N$-linear mappings (BPBpp for $N$-linear mappings, for short) if given $\varepsilon>0$, there exists $\eta(\varepsilon)>0$ such that whenever $A \in \mathcal{L}\left(X_{1}, \ldots, X_{N} ; Y\right)$ with $\|A\|=1$ and $\left(x_{1}, \ldots, x_{N}\right) \in S_{X_{1}} \times \cdots \times S_{X_{N}}$ satisfy

$$
\left\|A\left(x_{1}, \ldots, x_{N}\right)\right\|>1-\eta(\varepsilon)
$$

there is $B \in \mathcal{L}\left(X_{1}, \ldots, X_{N} ; Y\right)$ with $\|B\|=1$ such that

$$
\left\|B\left(x_{1}, \ldots, x_{N}\right)\right\|=1 \text { and }\|B-A\|<\varepsilon .
$$

Clearly, the BPBpp implies the BPBp but the converse is not true in general. Actually, if the pair $(X ; Y)$ has the BPBpp for some Banach space $Y$, then $X$ must be uniformly smooth (see [18, Proposition 2.3]). Also, it was proved in [18] that the pair $(X ; \mathbb{K})$ has the BPBpp if and only if $X$ is uniformly smooth. In both papers $[18,19]$ the authors presented such differences between these two properties and found many positive examples having BPBpp.

On the other hand, one may think about a "dual" version of the BPBpp where, instead of fixing the point, we fix the operator. 
Definition $1.3([16,21])$. We say that $\left(X_{1}, \ldots, X_{N} ; Y\right)$ has the Bishop-Phelps-Bollobás operator property for $N$-linear mappings (BPBop for $N$-linear mappings, for short) if given $\varepsilon>0$, there exists $\eta(\varepsilon)>0$ such that whenever $A \in \mathcal{L}\left(X_{1}, \ldots, X_{N} ; Y\right)$ with $\|A\|=1$ and $\left(x_{1}, \ldots, x_{N}\right) \in S_{X_{1}} \times \cdots \times S_{X_{N}}$ satisfy

$$
\left\|A\left(x_{1}, \ldots, x_{N}\right)\right\|>1-\eta(\varepsilon)
$$

there is $\left(z_{1}, \ldots, z_{N}\right) \in S_{X_{1}} \times \cdots \times S_{X_{N}}$ such that

$$
\left\|A\left(z_{1}, \ldots, z_{N}\right)\right\|=1 \text { and } \max _{1 \leqslant j \leqslant N}\left\|x_{j}-z_{j}\right\|<\varepsilon
$$

It was proved in $[29]$ that the pair $(X ; \mathbb{K})$ has the BPBop if and only $X$ is uniformly convex. So, in the scalar-valued case, these two properties are dual from each other; that is, $(X ; \mathbb{K})$ has the BPBpp if and only if $\left(X^{*} ; \mathbb{K}\right)$ has the BPBop. Nevertheless, it is known that there is no version for bounded linear operators of this property. Indeed, in [20], it is proved that for $\operatorname{dim}(X), \operatorname{dim}(Y) \geqslant 2$, the pair $(X ; Y)$ always fails the BPBop. Hence, there is no hope for this "uniform" property, which lead us to consider a "local type" of it as in $[16,33,34]$. In these papers, the function $\eta$ in the definition of the BPBop depends not only on $\varepsilon$ but also on a fixed norm one operator $T$, and some positive results are obtained, which are different from the uniform case when $\eta$ depends just on $\varepsilon$.

This motivated the current authors to study, in [21], all of the aforementioned properties in this local sense. In the paper, local versions of the BPBpp and BPBop (and also the BPBp) were addressed for linear operators. We give the precise definitions for $n$-linear mappings in section 2 . It turned out that these local properties are quite different from the corresponding uniform ones, as in the case of the BPBop (see [21, Section 5]). For instance, there is a connection between those properties and the subdifferentiability of the norm of the spaces (see [21, Theorem 2.3]). For the "local BPBpp", $\eta$ depends on a point $x \in S_{X}$ and $\varepsilon>0$, we have the following results.

Theorem $1.4([21])$. Consider the following pairs of Banach spaces $(X ; \mathbb{K})$ when $X$ is

(a) $c_{0}$ or

(b) the predual of Lorentz sequence space $d_{*}(w, 1)$ or

(c) the space $V M O$ (which is the predual of the Hardy space $H^{1}$ ) or

(d) a finite dimensional space,

and also the following pairs

(e) $\left(\ell_{1}^{N} ; L_{p}(\mu)\right)$ for $1<p<\infty, N \in \mathbb{N}$, and

(f) $\left(c_{0} ; L_{p}(\mu)\right)$ for $1 \leqslant p<\infty$.

Then, all of them satisfy this "local BPBpp".

In this paper we continue the study of these local properties, emphasizing in the multilinear setting. Following the notation in [21], we use the symbol $\mathbf{L}_{p, p}$ for the "local BPBpp", when $\eta$ depends on a point $x \in S_{X}$, and $\mathbf{L}_{o, o}$ for the "local BPBop", when $\eta$ depends on an operator $T \in S_{\mathcal{L}(X, Y)}$ (see Definition 2.1 below). In the next section, we give the proper definitions and first results. Among others, we obtain the following results (see Proposition 2.3 and the comment below Corollary 2.5).

- If $\left(X_{1}, \ldots, X_{N} ; Y\right)$ has property $\mathbf{L}_{p, p}\left(\right.$ or $\left.\mathbf{L}_{o, o}\right)$, then so does $\left(X_{i} ; \mathbb{K}\right)$ for every $1 \leqslant i \leqslant N$.

- There exist (finite dimensional) Banach spaces $X_{1}, \ldots, X_{N}, Y$ such that $\left(X_{1}, \ldots, X_{N} ; Y\right)$ has the $\mathbf{L}_{p, p}$ (respectively, $\mathbf{L}_{o, o}$ ) but fails the BPBpp (respectively, BPBop).

We also focus on the bilinear case when the domains are $\ell_{p}$-spaces. In that sense, we obtain the following results (see Theorem 2.7 and Remark 2.9).

- If $2<p, q<\infty$, then $\left(\ell_{p}, \ell_{q} ; \mathbb{K}\right)$ has the $\mathbf{L}_{p, p}$.

- If $1<p, q<\infty$, then $\left(\ell_{p}, \ell_{q} ; \mathbb{K}\right)$ has the $\mathbf{L}_{o, o}$ if and only if $p q>p+q$. Hence, there exist spaces $\ell_{p}, \ell_{q}$ such that $\left(\ell_{p}, \ell_{q} ; \mathbb{K}\right)$ fails the bilinear $\mathbf{L}_{o, o}$, while $\left(\ell_{p} ; \mathbb{K}\right)$ and $\left(\ell_{q} ; \mathbb{K}\right)$ have the linear $\mathbf{L}_{o, o}$ since both are uniformly smooth. 
In the proof of Theorem 2.7 we use a tensor product to prove that $\left(\ell_{p}, \ell_{q} ; \mathbb{K}\right)$ has the $\mathbf{L}_{p, p}$ for $2<p, q<$ $\infty$. As a consequence, we show that the norm of $\ell_{p} \hat{\otimes}_{\pi} \ell_{q}$ is strongly subdifferentiable for $2<p, q<\infty$. However if $p^{-1}+q^{-1} \geqslant 1$ or one of the indices $p, q$ takes the value 1 or $\infty$, then its norm is not strongly subdifferentiable. In Section 3, motivated by the geometric property approximate hyperplane series property (AHSP, for short) in [3, 4], we get a characterization of strong subdifferentiability. The AHSP characterizes a Banach space $Y$ for which $\left(\ell_{1} ; Y\right)$ and $\left(\ell_{1}, Y ; \mathbb{K}\right)$ have the BPBp (in the linear and bilinear case, respectively). Although the pairs $\left(\ell_{1} ; Y\right)$ and $\left(\ell_{1}, Y ; \mathbb{K}\right)$ do not have the $\mathbf{L}_{p, p}$ (since $\ell_{1}$ is not $\left.\mathrm{SSD}\right)$, we may ask if $\left(\ell_{1}^{N} ; Y\right)$ and $\left(\ell_{1}^{N}, Y ; \mathbb{K}\right)$ have it. In Proposition 3.2 we prove that the strong subdifferentiability of the norm of a Banach space $Y$ is equivalent to such characterization. As a consequence of this characterization, we prove that $\left(\ell_{1}^{N}, Y ; \mathbb{K}\right)$ has the $\mathbf{L}_{p, p}$ for bilinear forms if and only if the norm of a Banach space $Y$ is strongly subdifferentiable. Using similar ideas, we characterize the pairs $\left(\ell_{1}^{N} ; Y\right)$ having the $\mathbf{L}_{p, p}$ for operators, generalizing Theorem 1.4.(e). As a consequence of this last characterization, we prove that if a family $\left\{y_{\alpha}\right\}_{\alpha} \subset S_{Y}$ is uniformly strongly exposed with corresponding functionals $\left\{f_{\alpha}\right\}_{\alpha} \subset S_{Y^{*}}$, then $\left(\ell_{1}^{N} ; Y\right)$ has the $\mathbf{L}_{p, p}$ for operators whenever $\left\{f_{\alpha}\right\}_{\alpha}$ is a norming subset for the Banach space $Y$.

\section{The $\mathbf{L}_{p, p}$ AND THE $\mathbf{L}_{o, o}$ FOR $N$-LINEAR MAPPINGS}

We start this section by giving the precise definitions of the local Bishop-Phelps-Bollobás properties for $N$-linear mappings. These are the analogous of [21, Definition 2.1].

Definition 2.1. (a) We say that $\left(X_{1}, \ldots, X_{N} ; Y\right)$ has the $\mathbf{L}_{p, p}$ if given $\varepsilon>0$ and $\left(x_{1}, \ldots, x_{N}\right) \in$ $S_{X_{1}} \times \cdots \times S_{X_{N}}$, there is $\eta\left(\varepsilon,\left(x_{1}, \ldots, x_{N}\right)\right)>0$ such that whenever $A \in \mathcal{L}\left(X_{1}, \ldots, X_{N} ; Y\right)$ with $\|A\|=1$ satisfies

$$
\left\|A\left(x_{1}, \ldots, x_{N}\right)\right\|>1-\eta\left(\varepsilon,\left(x_{1}, \ldots, x_{N}\right)\right),
$$

there is $B \in \mathcal{L}\left(X_{1}, \ldots, X_{N} ; Y\right)$ with $\|B\|=1$ such that

$$
\left\|B\left(x_{1}, \ldots, x_{N}\right)\right\|=1 \text { and }\|B-A\|<\varepsilon .
$$

(b) We say that $\left(X_{1}, \ldots, X_{N} ; Y\right)$ has the $\mathbf{L}_{o, o}$ if given $\varepsilon>0$ and $A \in \mathcal{L}\left(X_{1}, \ldots, X_{N} ; Y\right)$ with $\|A\|=1$, there is $\eta(\varepsilon, A)>0$ such that whenever $\left(x_{1}, \ldots, x_{N}\right) \in S_{X_{1}} \times \cdots \times S_{X_{N}}$ satisfies

$$
\left\|A\left(x_{1}, \ldots, x_{N}\right)\right\|>1-\eta(\varepsilon, A),
$$

there is $\left(z_{1}, \ldots, z_{N}\right) \in S_{X_{1}} \times \cdots \times S_{X_{N}}$ such that

$$
\left\|A\left(z_{1}, \ldots, z_{N}\right)\right\|=1 \text { and } \max _{1 \leqslant j \leqslant N}\left\|x_{j}-z_{j}\right\|<\varepsilon .
$$

Let us observe that if $\left(X_{1}, \ldots, X_{N} ; Y\right)$ satisfies the $\mathbf{L}_{o, o}$, then every $A \in \mathcal{L}\left(X_{1}, \ldots, X_{N} ; Y\right)$ attains its norm and, consequently, all the Banach spaces $X_{i}$ 's must be reflexive. Indeed, if one of them is not reflexive, say $X_{k}$, by James theorem, there is $z_{k}^{*} \in S_{X_{k}^{*}}$ such that $\left|z_{k}^{*}\left(x_{k}\right)\right|<1$ for all $x_{k} \in S_{X_{k}}$. Now, taking arbitrary $y_{0} \in S_{Y}$ and $z_{i}^{*} \in S_{X_{i}^{*}}$ for each $i \neq k$ and defining $A \in \mathcal{L}\left(X_{1}, \ldots, X_{N} ; Y\right)$ by $A\left(x_{1}, \ldots, x_{N}\right):=\left(\Pi_{1 \leqslant i \leqslant N} z_{i}^{*}\left(x_{i}\right)\right) y_{0}$, we see that $A$ never attains its norm. Thus, in order to look for positive examples about the $\mathbf{L}_{o, o}$, we must assume, at least, that $X_{1}, \ldots, X_{N}$ are all reflexive Banach spaces.

It was proved in [16, Theorem 2.4] that if $X$ is a finite dimensional Banach space, then the pair $(X ; Y)$ has the $\mathbf{L}_{o, o}$ for every Banach space $Y$. By the similar proof, this can be generalized for $N$-linear mappings. However it does not hold for the $\mathbf{L}_{p, p}$ in general. Indeed, suppose that $Y$ is a strictly convex Banach space and that the pair $\left(\ell_{1}^{2} ; Y\right)$ has the $\mathbf{L}_{p, p}$. Then $Y$ is uniformly convex by [21, Proposition $3.2]$. So, choosing a strictly convex space $Y_{0}$ which is not uniformly convex, the pair $\left(\ell_{1}^{2}, Y_{0}\right)$ fails the $\mathbf{L}_{p, p}$ although $\ell_{1}^{2}$ is 2-dimensional. In the case that $Y$ is also finite dimensional, then we have a positive result as the following proposition. The proof is analogous to the operator case in [21, Proposition 2.8] and omitted.

Proposition 2.2. Let $N \in \mathbb{N}$ and let $X_{1}, \ldots, X_{N}$ be finite dimensional Banach spaces. Then, 
(a) $\left(X_{1}, \ldots, X_{N} ; Y\right)$ has the $\mathbf{L}_{o, o}$ for every Banach space $Y$;

(b) $\left(X_{1}, \ldots, X_{N} ; Y\right)$ has the $\mathbf{L}_{p, p}$ for every finite dimensional Banach space $Y$.

It is known that if the pair $(X ; Y)$ satisfies the BPBpp or the BPBop or the $\mathbf{L}_{p, p}$ for some Banach space $Y$, then so does $(X ; \mathbb{K})$ (see [16, Proposition 2.9], [18, Proposition 2.7] and [21, Proposition 2.7], respectively). The same happens with property $\mathbf{L}_{o, o}$. Indeed, given $\varepsilon>0$ and $x^{*} \in S_{X^{*}}$, we construct, for a fixed $y_{0} \in S_{Y}$, the operator $T \in \mathcal{L}(X, Y)$ given by $T(x):=x^{*}(x) y_{0}$ for all $x \in X$ and then we set $\eta\left(\varepsilon, x^{*}\right):=\eta(\varepsilon, T)>0$. If $x_{0} \in S_{X}$ is such that

$$
\left|x^{*}\left(x_{0}\right)\right|>1-\eta\left(\varepsilon, x^{*}\right),
$$

then $\left\|T\left(x_{0}\right)\right\|>1-\eta(\varepsilon, T)$. Thus, there is $x_{1} \in S_{X}$ such that

$$
\left\|T\left(x_{1}\right)\right\|=\left|x^{*}\left(x_{1}\right)\right|=1 \text { and }\left\|x_{1}-x_{0}\right\|<\varepsilon .
$$

Therefore, $(X, \mathbb{K})$ has the $\mathbf{L}_{o, o}$. By using the same arguments, we can extend those results for $N$-linear mappings. In the proof, we use the canonical isometry between $\mathcal{L}\left(X_{1}, \ldots, X_{N} ; \mathbb{K}\right)$ and $\mathcal{L}\left(X_{1}, \ldots, X_{N-1} ; X_{N}^{*}\right)$ to deduce item (b) below.

Proposition 2.3. Let $\mathcal{P}$ be one of the properties BPBpp, BPBop, $\mathbf{L}_{o, o}$ or $\mathbf{L}_{p, p}$.

(a) If $\left(X_{1}, \ldots, X_{N} ; Y\right)$ has the property $\mathcal{P}$, then so does $\left(X_{1}, \ldots, X_{N} ; \mathbb{K}\right)$.

(b) If $\left(X_{1}, \ldots, X_{N} ; \mathbb{K}\right)$ has the property $\mathcal{P}$ and $\mathcal{P}$ is not $\mathbf{L}_{p, p}$, then so does $\left(X_{1}, \ldots, X_{N-1} ; X_{N}^{*}\right)$.

(c) If $\left(X_{1}, \ldots, X_{N} ; Y\right)$ has the property $\mathcal{P}$, then so does $\left(X_{i} ; \mathbb{K}\right)$ for every $1 \leqslant i \leqslant N$.

Proof. The proof of (a) and (b) is sketched above. To prove (c), it suffices to show that the pair $\left(X_{1} ; \mathbb{K}\right)$ has property $\mathcal{P}$ whenever $\left(X_{1}, \ldots, X_{N} ; Y\right)$ does. Suppose first that $\mathcal{P}$ is not $\mathbf{L}_{p, p}$. Then, by item (a), we have that $\left(X_{1}, \ldots, X_{N} ; \mathbb{K}\right)$ has property $\mathcal{P}$ and, in virtue of $(\mathrm{b}),\left(X_{1}, \ldots, X_{N-1} ; X_{N}^{*}\right)$ does. Applying (a) again, we see that $\left(X_{1}, \ldots, X_{N-1} ; \mathbb{K}\right)$ has property $\mathcal{P}$. That is, if $\left(X_{1}, \ldots, X_{N} ; \mathbb{K}\right)$ has property $\mathcal{P}$, then $\left(X_{1}, \ldots, X_{N-1} ; \mathbb{K}\right)$ has property $\mathcal{P}$. Repeating this argument $(N-1)$-times, we see that $\left(X_{1} ; \mathbb{K}\right)$ has property $\mathcal{P}$.

Now, suppose that $\left(X_{1}, \ldots, X_{N} ; Y\right)$ has property $\mathbf{L}_{p, p}$. Then, by (a), we have that $\left(X_{1}, \ldots, X_{N} ; \mathbb{K}\right)$ has property $\mathbf{L}_{p, p}$. Given $\varepsilon>0$ and $x_{1}^{0} \in S_{X_{1}}$, we want to see that there is $\eta\left(\varepsilon, x_{1}^{0}\right)>0$ satisfying the definition of property $\mathbf{L}_{p, p}$ for the pair $\left(X_{1} ; \mathbb{K}\right)$. Consider $\left(x_{2}^{0}, \ldots, x_{N}^{0}\right) \in S_{X_{2}} \times \cdots \times S_{X_{N}}$ and $\left(x_{2}^{*}, \ldots, x_{N}^{*}\right) \in$ $S_{X_{2}^{*}} \times \cdots \times S_{X_{N}^{*}}$ such that $x_{i}^{*}\left(x_{i}^{0}\right)=1$, for $i=2, \ldots, N$, and put $\eta\left(\varepsilon, x_{1}^{0}\right):=\eta\left(\varepsilon,\left(x_{1}^{0}, \ldots, x_{N}^{0}\right)\right)$, which exists by hypothesis. Suppose that $x_{1}^{*} \in S_{X_{1}^{*}}$ is such that $\left|x_{1}^{*}\left(x_{1}^{0}\right)\right|>1-\eta\left(\varepsilon, x_{1}^{0}\right)$. Then, defining $A\left(x_{1}, \ldots, x_{N}\right)=x_{1}^{*}\left(x_{1}\right) x_{2}^{*}\left(x_{2}\right) \cdots x_{N}^{*}\left(x_{N}\right)$, we have that $A \in \mathcal{L}\left(X_{1}, \ldots, X_{N} ; \mathbb{K}\right),\|A\|=1$, and

$$
\left|A\left(x_{1}^{0}, \ldots, x_{N}^{0}\right)\right|>1-\eta\left(\varepsilon,\left(x_{1}^{0}, \ldots, x_{N}^{0}\right)\right) .
$$

Consequently, there exists $B \in \mathcal{L}\left(X_{1}, \ldots, X_{N} ; \mathbb{K}\right)$ with $\|B\|=1$ such that $\left|B\left(x_{1}^{0}, \ldots, x_{N}^{0}\right)\right|=1$ and $\| B-$ $A \|<\varepsilon$. Therefore, defining $y_{1}^{*} \in X_{1}^{*}$ by $y_{1}^{*}(\cdot)=B\left(\cdot, x_{2}^{0}, \ldots, x_{N}^{0}\right)$, we see that

$$
y_{1}^{*} \in S_{X_{1}^{*}}, \quad\left|y_{1}^{*}\left(x_{1}^{0}\right)\right|=1, \quad \text { and } \quad\left\|y_{1}^{*}-x_{1}^{*}\right\| \leqslant\|B-A\|<\varepsilon,
$$

which is the desired statement.

The item (b) above does not hold for the $\mathbf{L}_{p, p}$; we provide a counterexample in Remark 3.4.

Recall that the norm of a Banach space $X$ is said to be strongly subdifferentiable (SSD, for short) at $x \in S_{X}$ if the one-sided limit

$$
\lim _{t \rightarrow 0^{+}} \frac{\|x+t h\|-\|x\|}{t}
$$

exists uniformly for $h \in B_{X}$. If (1) holds for every element in the unit sphere $S_{X}$, we say that the norm of $X$ is SSD or just $X$ is SSD. This differentiability is known to be strictly weaker than Fréchet differentiability. By the characterization of SSD due to C. Franchetti and R. Payá (see [23, Theorem $1.2])$, we have that $(X ; \mathbb{K})$ has the $\mathbf{L}_{p, p}$ if and only if the norm of $X$ is SSD and, by duality, $(X ; \mathbb{K})$ has the $\mathbf{L}_{o, o}$ if and only if $X$ is reflexive and the norm of $X^{*}$ is SSD (see [21, Theorem 2.3] and also [26] where this fact was already observed). 
By using this result and the characterization of property BPBpp for the pair $(X ; \mathbb{K})$ given in $[18$, Proposition 2.1], we have the following consequences of Proposition 2.3.

Corollary 2.4. Let $N \in \mathbb{N}$ and $X_{1}, \ldots, X_{N}$ be Banach spaces.

(a) If $\left(X_{1}, \ldots, X_{N} ; Y\right)$ has the BPBpp for some Banach space $Y$, then $X_{i}$ is uniformly smooth for each $i=1, \ldots, N$.

(b) If $\left(X_{1}, \ldots, X_{N} ; Y\right)$ has the $\mathbf{L}_{p, p}$ for some Banach space $Y$, then $X_{i}$ is $\operatorname{SSD}$ for each $i=1, \ldots, N$.

Another consequence of Proposition 2.3 is that, for spaces of dimension greater than 2, there is no BPBop for bilinear mappings. Indeed, if $\operatorname{dim}(X), \operatorname{dim}(Y) \geqslant 2$ and $(X, Y ; Z)$ has the BPBop for some Banach space $Z$, then by Proposition 2.3, the pair $\left(X, Y^{*}\right)$ has the BPBop for operators and, as we already mentioned in the Introduction, this is not possible. We can deduce the same for $N$-linear mappings.

Corollary 2.5. Let $N \in \mathbb{N}$. Let $X_{i}$ be a Banach space with $\operatorname{dim}\left(X_{i}\right) \geqslant 2$ for $1 \leqslant i \leqslant N$. Then, $\left(X_{1}, \ldots, X_{N} ; Y\right)$ fails the BPBop for every Banach space $Y$.

At this point, we can point out some differences between properties BPBpp (respectively, BPBop) and $\mathbf{L}_{p, p}$ (respectively, $\mathbf{L}_{o, o}$ ). For instance, if $X_{i}=\ell_{1}^{2}$ or $\ell_{\infty}^{2}$ and $Y$ is any finite dimensional Banach space, then by Proposition 2.2 we have that $\left(X_{1}, \cdots, X_{N} ; Y\right)$ has the $\mathbf{L}_{p, p}$ (respectively, $\left.\mathbf{L}_{o, o}\right)$ while, in virtue of Corollary 2.4.(a) (respectively, Corollary 2.5) it fails property BPBpp (respectively, BPBop).

Next we focus on the bilinear case when the domains are $\ell_{p}$-spaces. For the part (b) of Theorem 2.7 below we need the following lemma, which gives a converse of Proposition 2.3 (b) for property $\mathbf{L}_{o, o}$.

Lemma 2.6. Let $X, Y$ be Banach spaces and suppose that $Y$ is uniformly convex. Then $(X, Y ; \mathbb{K})$ has the $\mathbf{L}_{o, o}$ for bilinear forms if and only if the pair $\left(X ; Y^{*}\right)$ has the $\mathbf{L}_{o, o}$ for operators.

Proof. From Proposition $2.3(\mathrm{~b})$, if $(X, Y ; \mathbb{K})$ has the $\mathbf{L}_{o, o}$ then so does $\left(X ; Y^{*}\right)$. Hence, we only have to prove the converse. Let $\varepsilon \in(0,1)$ be given. Since $Y$ is uniformly convex, the pair $(Y ; \mathbb{K})$ has the BPBop with some $\tilde{\eta}(\varepsilon)>0$ (see [29, Theorem 2.1]). This means that if $y^{*} \in S_{Y^{*}}$ and $y \in B_{Y}$ satisfy $\left|y^{*}(y)\right|>1-\tilde{\eta}(\varepsilon)$, then, there exists $z \in S_{Y}$ such that $\left|y^{*}(z)\right|=1$ and $\|y-z\|<\varepsilon$. Fix $A \in \mathcal{L}(X, Y ; \mathbb{K})$ with $\|A\|=1$ and take its associated operator $T_{A} \in S_{\mathcal{L}\left(X, Y^{*}\right)}$. Consider $\xi>0$ to be such that $2 \xi<\min \{\tilde{\eta}(\varepsilon), \varepsilon\}$ and set

$$
\eta(\varepsilon, A):=\min \left\{\xi, \eta^{\prime}\left(\xi, T_{A}\right)\right\}>0,
$$

where $\eta^{\prime}$ is the function in the definition of $\mathbf{L}_{o, o}$ for the pair $\left(X ; Y^{*}\right)$. Let $\left(x_{0}, y_{0}\right) \in S_{X} \times S_{Y}$ be such that

Then, since

$$
\left|A\left(x_{0}, y_{0}\right)\right|>1-\eta(\varepsilon, A)
$$

there is $x_{1} \in S_{X}$ such that

$$
\left\|T_{A}\left(x_{1}\right)\right\|_{Y^{*}}=1 \text { and }\left\|x_{1}-x_{0}\right\|<\xi<\varepsilon .
$$

Now, since $T_{A}\left(x_{1}\right) \in S_{Y^{*}}$ and $y_{0} \in S_{Y}$ satisfy

$$
\begin{aligned}
\left|\left[T_{A}\left(x_{1}\right)\right]\left(y_{0}\right)\right| & \geqslant\left|T_{A}\left(x_{0}\right)\left(y_{0}\right)\right|-\left|T_{A}\left(x_{1}-x_{0}\right)\left(y_{0}\right)\right| \\
& \geqslant\left|A\left(x_{0}, y_{0}\right)\right|-\left\|x_{1}-x_{0}\right\| \\
& >1-\eta(\varepsilon, A)-\xi \\
& >1-2 \xi>1-\tilde{\eta}(\varepsilon),
\end{aligned}
$$

there is $y_{1} \in S_{Y}$ such that $\left|\left[T_{A}\left(x_{1}\right)\right]\left(y_{1}\right)\right|=1$ and $\left\|y_{1}-y_{0}\right\|<\varepsilon$. Since

$$
1=\left|\left[T_{A}\left(x_{1}\right)\right]\left(y_{1}\right)\right|=\left|A\left(x_{1}, y_{1}\right)\right|, \quad\left\|x_{1}-x_{0}\right\|<\varepsilon, \text { and }\left\|y_{1}-y_{0}\right\|<\varepsilon,
$$

we have proved that $(X, Y ; \mathbb{K})$ has the $\mathbf{L}_{o, o}$ for bilinear forms, as desired. 
Denote by $X \hat{\otimes}_{\pi} Y$ the projective tensor product of the Banach spaces $X$ and $Y$. Recall that the space $\mathcal{L}(X, Y ; Z)$ is isometrically isomorphic to $\mathcal{L}\left(X \hat{\otimes}_{\pi} Y ; Z\right)$ (see, for example, [32, Theorem 2.9]). Recall also the following definition: a dual Banach space $X^{*}$ has the $w^{*}$-Kadec-Klee property if $\left\|x_{\alpha}-x\right\| \rightarrow 0$ whenever $\left\|x_{\alpha}\right\| \rightarrow\|x\|$ and $x_{\alpha} \stackrel{w^{*}}{\longrightarrow} x$. If this holds for sequences, we say that $X^{*}$ has the sequential $w^{*}$-Kadec-Klee property. For some background concerning these properties, see [11, 27]. It is worth mentioning that if the unit ball $B_{X^{*}}$ is $w^{*}$-sequentially compact, then the sequential $w^{*}$-Kadec-Klee property implies the $w^{*}$-Kadec-Klee property on $X^{*}$ (see [11, Proposition 1.4]). Now, we prove the desired result.

Theorem 2.7. For $1<s<\infty$, let $s^{\prime}$ be the conjugate of $s$ (that is, $\frac{1}{s}+\frac{1}{s^{\prime}}=1$ ).

(a) If $2<p, q<\infty$, then $\left(\ell_{p}, \ell_{q} ; \mathbb{K}\right)$ has the $\mathbf{L}_{p, p}$.

(b) If $1<p, q<\infty$, then $\left(\ell_{p}, \ell_{q} ; \mathbb{K}\right)$ has the $\mathbf{L}_{o, o}$ if and only if $p q>p+q$ (or, equivalently, $p>q^{\prime}$ ).

Proof. (a) It is known that if $X^{*}$ has the $w^{*}$-Kadec-Klee property, then the pair $(X, \mathbb{K})$ has the $\mathbf{L}_{p, p}$ (see [21, Proposition 2.6]). On the other hand, in [22, Theorem 4] it was proved that if $1<r<2<s<\infty$, then $\mathcal{L}\left(\ell_{s} ; \ell_{r}\right)=\mathcal{L}\left(\ell_{s}, \ell_{r^{\prime}} ; \mathbb{K}\right)=\left(\ell_{s} \hat{\otimes}_{\pi} \ell_{r^{\prime}}\right)^{*}$ has the sequential $w^{*}$-uniform-Kadec-Klee property, which implies the sequential $w^{*}$-Kadec-Klee property. Indeed, since $\ell_{s} \hat{\otimes}_{\pi} \ell_{r^{\prime}}$ is reflexive (see, for instance, [32, Corollary 4.24]), then its unit dual ball is $w^{*}$-sequentially compact and, consequently, $\left(\ell_{s} \hat{\otimes}_{\pi} \ell_{r^{\prime}}\right)^{*}$ has the $w^{*}$-Kadec-Klee property. Hence, the pair $\left(\ell_{p} \hat{\otimes}_{\pi} \ell_{q} ; \mathbb{K}\right)$ has the $\mathbf{L}_{p, p}$ for $2<p, q<\infty$.

For a given $\varepsilon>0$ and a fixed norm-one point $(x, y) \in S_{\ell_{p}} \times S_{\ell_{q}}$, consider $\eta(\varepsilon, x \otimes y)>0$ to be the function in the definition of $\mathbf{L}_{p, p}$ for the pair $\left(\ell_{p} \hat{\otimes}_{\pi} \ell_{q} ; \mathbb{K}\right)$. Let $A \in \mathcal{L}\left(\ell_{p}, \ell_{q} ; \mathbb{K}\right)$ with $\|A\|=1$ be such that

$$
|A(x, y)|>1-\eta(\varepsilon, x \otimes y) \text {. }
$$

Consider $\hat{A}$ to be the corresponding element in $S_{\left(\ell_{p} \hat{\otimes}_{\pi} \ell_{q}\right) *}$ via the canonical isometry. Then, we have

$$
|\hat{A}(x \otimes y)|=|A(x, y)|>1-\eta(\varepsilon, x \otimes y) .
$$

Since the pair $\left(\ell_{p} \hat{\otimes}_{\pi} \ell_{q} ; \mathbb{K}\right)$ has the $\mathbf{L}_{p, p}$ with $\eta(\varepsilon, x \otimes y)>0$, there exists $\hat{B} \in S_{\left(\ell_{p} \hat{\otimes}_{\pi} \ell_{q}\right)^{*}}$ such that

$$
|\hat{B}(x \otimes y)|=1 \quad \text { and } \quad\|\hat{B}-\hat{A}\|<\varepsilon .
$$

Now we take $B \in S_{\mathcal{L}\left(\ell_{p}, \ell_{q} ; \mathbb{K}\right)}$, the corresponding element to $\hat{B}$ via the canonical isometry. Then, $|B(x, y)|=$ $|\hat{B}(x \otimes y)|=1$ and $\|B-A\|=\|\hat{B}-\hat{A}\|<\varepsilon$. This proves (a).

(b) Let $1<p, q<\infty$. By Lemma 2.6, $\left(\ell_{p}, \ell_{q} ; \mathbb{K}\right)$ has the $\mathbf{L}_{o, o}$ if and only if $\left(\ell_{p} ; \ell_{q^{\prime}}\right)$ has the $\mathbf{L}_{o, o}$ and, in virtue of [16, Theorem 2.21], this happens if and only if $p>q^{\prime}$.

Note that inside the proof of Theorem 2.7, we have proved that the pair $\left(\ell_{p} \hat{\otimes}_{\pi} \ell_{q} ; \mathbb{K}\right)$ has the $\mathbf{L}_{p, p}$ for $2<p, q<\infty$. This yields to the following consequence.

Corollary 2.8. For $p, q \geqslant 1$

(a) if $2<p, q<\infty$, then the norm of $\ell_{p} \hat{\otimes}_{\pi} \ell_{q}$ is $\mathrm{SSD}$.

(b) if $p^{-1}+q^{-1} \geqslant 1$ or one of $p$ and $q$ is 1 or $\infty$, then the norm of $\ell_{p} \hat{\otimes}_{\pi} \ell_{q}$ is not SSD.

Proof. As we already mentioned, item (a) follows from the proof of Theorem 2.7 and [21, Theorem 2.3]. To prove (b), note that if $p^{-1}+q^{-1} \geqslant 1$, then the main diagonal $\mathcal{D}=\overline{\operatorname{span}\left\{e_{n} \otimes e_{n}: n \in \mathbb{N}\right\}}$ is onecomplemented in $\ell_{p} \hat{\otimes}_{\pi} \ell_{q}$ and isometrically isomorphic to $\ell_{1}$ (see, for instance, [6, Theorem 1.3]). Hence, if the norm of $\ell_{p} \hat{\otimes}_{\pi} \ell_{q}$ were SSD, by [21, Theorem 2.3] we would have that $\left(\ell_{p} \hat{\otimes}_{\pi} \ell_{q}, \mathbb{K}\right)$ has the $\mathbf{L}_{p, p}$ and, by $[21$, Proposition $4.4(\mathrm{~b})],\left(\ell_{1}, \mathbb{K}\right)$ would have the $\mathbf{L}_{p, p}$, which gives the desired contradiction. Suppose now that $p$ or $q$ take the value 1 or $\infty$. As we showed in the proof of Theorem 2.7 , if $\ell_{p} \hat{\otimes}_{\pi} \ell_{q}$ were SSD then $\left(\ell_{p}, \ell_{q} ; \mathbb{K}\right)$ would have the $\mathbf{L}_{p, p}$ for bilinear forms, which is not possible by Proposition 2.3.(c) since neither $\ell_{1}$ nor $\ell_{\infty}$ are not SSD. 
In the proof of Theorem 2.7 we showed that if the pair $\left(\ell_{p} \hat{\otimes}_{\pi} \ell_{q} ; \mathbb{K}\right)$ has the $\mathbf{L}_{p, p}$ (or, equivalently, $\ell_{p} \hat{\otimes}_{\pi} \ell_{q}$ is SSD) then $\left(\ell_{p}, \ell_{q} ; \mathbb{K}\right)$ has the $\mathbf{L}_{p, p}$ for bilinear forms. However, it is worth to remark that the converse is not true. For instance, $\left(\ell_{2}, \ell_{2} ; \mathbb{K}\right)$ has the $\mathbf{L}_{p, p}$ for bilinear forms (moreover, it has the BPBpp by $\left[18\right.$, Corollary 3.2]) but $\ell_{2} \hat{\otimes}_{\pi} \ell_{2}$ is not SSD.

We finish this section with some remarks and open questions.

Remark 2.9. (a) Since the uniform properties imply the local properties, when trying to prove that $\left(X_{1}, \ldots, X_{N} ; \mathbb{K}\right)$ has the $\mathbf{L}_{p, p}$ (respectively, $\mathbf{L}_{o, o}$ ) for some Banach spaces $X_{1}, \ldots, X_{N}$, it is natural to ask first if $\left(X_{1}, \ldots, X_{N} ; \mathbb{K}\right)$ has (or not) the BPBpp (respectively, BPBop). Taking into account Theorem 2.7 we must say that, to the best of our knowledge, it is not known whether $\left(\ell_{p}, \ell_{q} ; \mathbb{K}\right)$ has the BPBpp when $2<p, q<\infty$. On the other hand, by Corollary $2.5,\left(\ell_{p}, \ell_{q} ; \mathbb{K}\right)$ fails the BPBop for every $1<p, q<\infty$.

(b) By Proposition 2.3 we know that if $\left(X_{1}, \ldots, X_{N} ; \mathbb{K}\right)$ has the $\mathbf{L}_{p, p}$ (respectively, $\mathbf{L}_{o, o}$ ), then so does $\left(X_{i} ; \mathbb{K}\right)$ for $1 \leqslant i \leqslant N$. Hence, we may ask if $\left(X_{1}, \ldots, X_{N} ; \mathbb{K}\right)$ has one of the mentioned properties whenever the pairs $\left(X_{i} ; \mathbb{K}\right)$ does. In that sense, note that $\left(\ell_{p} ; \mathbb{K}\right)$ and $\left(\ell_{q} ; \mathbb{K}\right)$ both have the $\mathbf{L}_{o, o}$ for every $1<p, q<\infty$ (since $\ell_{p}$ and $\ell_{q}$ are both reflexive and $\ell_{p}^{*}, \ell_{q}^{*}$ are both SSD) but, in virtue of Theorem 2.7 (b), there are $p, q$ such that $\left(\ell_{p}, \ell_{q} ; \mathbb{K}\right)$ fails the $\mathbf{L}_{o, o}$. We also have that the pairs $\left(\ell_{p} ; \mathbb{K}\right)$ and $\left(\ell_{q} ; \mathbb{K}\right)$ have the $\mathbf{L}_{p, p}$ for every $1<p, q<\infty$ but we do not know if there is some $1<p, q<\infty$ such that $\left(\ell_{p}, \ell_{q}\right.$; $\left.\mathbb{K}\right)$ fails the $\mathbf{L}_{p, p}$ for bilinear forms.

\section{LOCAL AHSP}

Our main aim in this section is to give a characterization for the Banach space $Y$ in such a way that $\left(\ell_{1}^{N}, Y ; \mathbb{K}\right)$ satisfies the $\mathbf{L}_{p, p}$. Indeed, we prove that the norm of a Banach space $Y$ is SSD if and only if $\left(\ell_{1}^{N}, Y ; \mathbb{K}\right)$ has the $\mathbf{L}_{p, p}$ for bilinear forms. To do so, we get a characterization of SSD that is motivated by the approximate hyperplane series property (AHSP, for short), which was defined for the first time in [3]. Before giving our characterization, we recall the definition and important results concerning this property.

Definition $3.1([3])$. A Banach space $Y$ has the AHSP if for every $\varepsilon>0$, there is $\eta(\varepsilon)>0$ such that given a sequence $\left(y_{k}\right) \subset S_{Y}$ and a convex series $\sum_{k=1}^{\infty} \alpha_{k}$ such that

$$
\left\|\sum_{k=1}^{\infty} \alpha_{k} y_{k}\right\|>1-\eta(\varepsilon),
$$

there exist $A \subset \mathbb{N}, y^{*} \in S_{Y^{*}}$, and $\left\{z_{j}: j \in A\right\}$ satisfying the following conditions:

$$
\sum_{k \in A} \alpha_{k}>1-\varepsilon, \quad\left\|z_{k}-y_{k}\right\|<\varepsilon, \quad \text { and } \quad y^{*}\left(z_{k}\right)=1 \text { for every } k \in A
$$

Finite dimensional, uniformly convex and lush spaces are known examples of Banach spaces satisfying the AHSP (see [3, Propositions 3.5, 3.8] and [15, Theorem 7], respectively). More specifically, $L_{p}(\mu)$ spaces for arbitrary $1 \leqslant p \leqslant \infty$ and $C(K)$-spaces for a compact Hausdorff $K$ are concrete examples of such a Banach spaces. This property was defined in [3] in order to give a characterization for the Banach spaces $Y$ such that the pair $\left(\ell_{1} ; Y\right)$ has the BPBp for operators. Here, we are interested to get a local version of AHSP which is related with the $\mathbf{L}_{p, p}$ for bilinear mappings (see [4, Definition 3.1] and [17, Section 3] for AHSP for bilinear mappings). It turns out that this local version of AHSP is equivalent to SSD of the norm.

Proposition 3.2. Let $Y$ be a Banach space. For any $N \in \mathbb{N}$, the following are equivalent.

(a) The norm of $Y$ is SSD. 
(b) Given $\varepsilon>0$, a nonempty set $A \subset\{1, \ldots, N\},\left(\alpha_{j}\right)_{j \in A}$ with $\alpha_{j}>0$ for all $j \in A$ and $\sum_{j \in A} \alpha_{j}=1$, and $y \in S_{Y}$, there is $\eta=\eta\left(\varepsilon,\left(\alpha_{j}\right)_{j \in A}, y\right)>0$ such that whenever $\left(y_{j}^{*}\right)_{j \in A} \subset S_{Y^{*}}$ satisfies

$$
\operatorname{Re} \sum_{j \in A} \alpha_{j} y_{j}^{*}(y)>1-\eta
$$

there is $\left(z_{j}^{*}\right)_{j \in A} \subset S_{Y^{*}}$ such that

$$
z_{j}^{*}(y)=1 \quad \text { and } \quad\left\|z_{j}^{*}-y_{j}^{*}\right\|<\varepsilon,
$$

for all $j \in A$.

Proof. (b) implies (a) by considering a singleton $A$ and recalling that $Y$ is SSD if and only if $(Y, \mathbb{K})$ has the $\mathbf{L}_{p, p}$. Now assume that the norm of $Y$ is SSD or, equivalently, that the pair $(Y ; \mathbb{K})$ has the $\mathbf{L}_{p, p}$ with some function $\eta^{\prime}$. Fix $\varepsilon>0$, a nonempty set $A \subset\{1, \ldots, N\},\left(\alpha_{j}\right)_{j \in A}$ with $\alpha_{j}>0$ for all $j \in A$ and $\sum_{j \in A} \alpha_{j}=1$, and $y \in S_{Y}$. Set $\alpha:=\min _{j \in A} \alpha_{j}$ and

$$
\eta\left(\varepsilon,\left(\alpha_{j}\right)_{j \in A}, y\right):=\alpha \eta^{\prime}\left(\frac{\varepsilon^{2}}{16}, y\right)>0 .
$$

Note that we may assume that $\eta^{\prime}(\varepsilon, y) \leqslant \varepsilon$ for every $\varepsilon>0$. Let $\left(y_{j}^{*}\right)_{j \in A} \subset S_{Y^{*}}$ be such that

$$
\operatorname{Re} \sum_{j \in A} \alpha_{j} y_{j}^{*}(y)>1-\eta\left(\varepsilon,\left(\alpha_{j}\right)_{j \in A}, y\right) .
$$

Then, for each $k \in A$, we have

$$
1-\alpha_{k} \eta^{\prime}(\varepsilon, y) \leqslant 1-\alpha \eta^{\prime}(\varepsilon, y)=1-\eta\left(\varepsilon,\left(\alpha_{j}\right)_{j \in A}, y\right)<\operatorname{Re} \sum_{j \in A} \alpha_{j} y_{j}^{*}(y) \leqslant \operatorname{Re} \alpha_{k} y_{k}^{*}(y)+\left(1-\alpha_{k}\right) .
$$

So,

$$
\operatorname{Re} y_{k}^{*}(y)>1-\eta^{\prime}\left(\frac{\varepsilon^{2}}{16}, y\right) \text { for each } k \in A \text {. }
$$

Since $(Y ; \mathbb{K})$ has the $\mathbf{L}_{p, p}$ with $\eta^{\prime}$, for each $k \in A$, there is $\tilde{z}_{k}^{*} \in S_{Y^{*}}$ such that

$$
\left|\tilde{z}_{k}^{*}(y)\right|=1 \quad \text { and } \quad\left\|\tilde{z}_{k}^{*}-y_{k}^{*}\right\|<\frac{\varepsilon^{2}}{16} .
$$

For each $k \in A$, write $\tilde{z}_{k}^{*}(y)=e^{i \theta_{k}}\left|\tilde{z}_{k}^{*}(y)\right|=e^{i \theta_{k}}$. Then,

$$
\left\|e^{-i \theta_{k}} \tilde{z}_{k}^{*}-y_{k}^{*}\right\| \leqslant\left|1-e^{i \theta_{k}}\right|+\left\|\tilde{z}_{k}^{*}-y_{k}^{*}\right\|
$$

for all $k \in A$. Now, note that whenever $k \in A$, we have

$$
\operatorname{Re} e^{i \theta_{k}}=\operatorname{Re} \tilde{z}_{k}^{*}(y) \geqslant \operatorname{Re} y_{k}^{*}(y)-\left\|\tilde{z}_{k}^{*}-y_{k}^{*}\right\|>1-\eta^{\prime}\left(\frac{\varepsilon^{2}}{16}, y\right)-\frac{\varepsilon^{2}}{16}>1-\frac{\varepsilon^{2}}{8} .
$$

So,

$$
\begin{aligned}
\left|1-e^{i \theta_{k}}\right|^{2} & =\left(\operatorname{Re} e^{i \theta_{k}}-1\right)^{2}+\left(\operatorname{Im} e^{i \theta_{k}}\right)^{2} \\
& =1-2 \operatorname{Re} e^{i \theta_{k}}+\left(\operatorname{Re} e^{i \theta_{k}}\right)^{2}+\left(\operatorname{Im} e^{i \theta_{k}}\right)^{2} \\
& =2\left(1-\operatorname{Re} e^{i \theta_{k}}\right)<\frac{\varepsilon^{2}}{4},
\end{aligned}
$$

which implies $\left|1-e^{i \theta_{k}}\right|<\frac{\varepsilon}{2}$ for every $k \in A$. Then, for each $k \in A$, we have

$$
\left\|e^{-i \theta_{k}} \tilde{z}_{k}^{*}-y_{k}^{*}\right\|<\frac{\varepsilon}{2}+\frac{\varepsilon^{2}}{16}<\varepsilon .
$$

Setting $z_{k}^{*}:=e^{-i \theta_{k}} \tilde{z}_{k}^{*}$ for each $k \in A$, we have that $z_{k}^{*}(y)=1$ and $\left\|z_{k}^{*}-y_{k}^{*}\right\|<\varepsilon$, which proves that (a) implies (b). 
Note that part (b) of Proposition 3.2 is a kind of local version of AHSP for the Bishop-Phelps-Bollobás point property since we do not move the initial point and also the $\eta$ in its definition depends not only on a positive $\varepsilon$ but also on a finite convex series and on a norm-one point. Observe that, by a simple change of parameters, we can take $\left(y_{j}^{*}\right)_{j \in A}$ in $B_{Y^{*}}$ instead of $S_{Y^{*}}$ in item (b) and we are using this fact without any explicit reference in the next theorem, where we prove the promised characterization of property $\mathbf{L}_{p, p}$ for $\left(\ell_{1}^{N}, Y ; \mathbb{K}\right)$.

Theorem 3.3. Let $Y$ be a Banach space and $N \in \mathbb{N}$. Then, $\left(\ell_{1}^{N}, Y ; \mathbb{K}\right)$ has the $\mathbf{L}_{p, p}$ if and only if the norm of $Y$ is SSD.

Proof. If $\left(\ell_{1}^{N}, Y ; \mathbb{K}\right)$ has the $\mathbf{L}_{p, p}$ for bilinear forms then, by Proposition 2.3 (c), the pair $(Y$; $\mathbb{K})$ has the $\mathbf{L}_{p, p}$, which is equivalent to say that the norm of $Y$ is SSD. Suppose now that the norm of $Y$ is SSD. Let $\varepsilon>0$ and $(x, y) \in S_{\ell_{1}^{N}} \times S_{Y}$ be given. We write $x=\left(x_{1}, \ldots, x_{N}\right)$ and assume that $x_{j} \geqslant 0$ for all $j=1, \ldots, N$ by composing it with an isometry if necessary. Let $A=\left\{j \in\{1, \ldots, N\}: x_{j} \neq 0\right\}$. Then $\|x\|_{1}=\sum_{j \in A} x_{j}=1$. Consider $\left(x_{j}\right)_{j \in A}$ and by Proposition 3.2, we may set

$$
\eta(\varepsilon,(x, y)):=\eta\left(\varepsilon,\left(x_{j}\right)_{j \in A}, y\right)>0 .
$$

Let $A \in \mathcal{L}\left(\ell_{1}^{N}, Y ; \mathbb{K}\right)$ with $\|A\|=1$ be such that

$$
|A(x, y)|>1-\eta(\varepsilon,(x, y)) .
$$

By rotating $A$, if necessary, we may assume that $\operatorname{Re} A(x, y)>1-\eta(\varepsilon,(x, y))$. So,

$$
\operatorname{Re} A(x, y)=\operatorname{Re} \sum_{j \in A} x_{j} A\left(e_{j}, y\right)>1-\eta(\varepsilon,(x, y)) .
$$

Define $y_{j}^{*}(y):=A\left(e_{j}, y\right)$ for every $y \in Y$. Since $\|A\|=1$, we have that $\left(y_{j}^{*}\right)_{j \in A} \subset B_{Y^{*}}$ and

$$
\operatorname{Re} \sum_{j \in A} x_{j} y_{j}^{*}(y)>1-\eta(\varepsilon,(x, y))=1-\eta\left(\varepsilon,\left(x_{j}\right)_{j \in A}, y\right) .
$$

By Proposition 3.2, there is $\left(z_{j}^{*}\right)_{j \in A} \subset S_{Y^{*}}$ such that $z_{j}^{*}(y)=1$ and $\left\|z_{j}^{*}-y_{j}^{*}\right\|<\varepsilon$, for all $j \in A$. Now, define $B: \ell_{1}^{N} \times Y \longrightarrow \mathbb{K}$ by

$$
B(u, v):=\sum_{j \in A} u_{j} z_{j}^{*}(v)+\sum_{j \notin A} u_{j} A\left(e_{j}, v\right),
$$

for $u=\left(u_{j}\right)_{j=1}^{N} \in \ell_{1}^{N}$ and $v \in Y$. So, $\|B\| \leqslant 1$ and

$$
|B(x, y)|=\left|\sum_{j \in A} x_{j} B\left(e_{j}, y\right)\right|=\left|\sum_{j \in A} x_{j} z_{j}^{*}(y)\right|=\sum_{j \in A} x_{j}=1 .
$$

Then $\|B\|=|B(x, y)|=1$. Also, for every $(u, v) \in S_{\ell_{1}^{N}} \times S_{Y}$, we have

$$
|B(u, v)-A(u, v)|=\left|\sum_{j \in A} u_{j}\left(z_{j}^{*}-y_{j}^{*}\right)(v)\right|<\varepsilon \sum_{j \in A} u_{j}\|v\| \leqslant \varepsilon .
$$

Therefore $\|B-A\|<\varepsilon$, and this shows that $\left(\ell_{1}^{N}, Y ; \mathbb{K}\right)$ has the $\mathbf{L}_{p, p}$ for bilinear forms.

Remark 3.4. We can use Theorem 3.3 to show that Proposition 2.3.(b) does not hold for $\mathbf{L}_{p, p}$. Consider a dual space $Y^{*}$ which is isomorphic to $\ell_{1}$ and its norm is locally uniformly rotund (and then strictly convex) [28]. Then, the norm of $Y$ is Fréchet differentiable (see, for example, [25, Fact 8.18]), and so it is SSD. By Theorem 3.3, we have that $\left(\ell_{1}^{N}, Y ; \mathbb{K}\right)$ has the $\mathbf{L}_{p, p}$. Suppose by contradiction that the pair $\left(\ell_{1}^{N} ; Y^{*}\right)$ has the $\mathbf{L}_{p, p}$ for operators. Since $\mathbf{L}_{p, p}$ is stable under one-complemented subspaces on the domain (see [21, Proposition 4.4]), the pair $\left(\ell_{1}^{2} ; Y^{*}\right)$ also has the $\mathbf{L}_{p, p}$. Since $Y^{*}$ is strictly convex, by using [21, Proposition 3.2] we get that $Y^{*}$ should be uniformly convex, which is not possible. So, $Y$ is the desired counterexample. 
Analogously to the bilinear case, we obtain a characterization of those Banach spaces $Y$ such that the pair $\left(\ell_{1}^{N} ; Y\right)$ has the $\mathbf{L}_{p, p}$ for operators for an arbitrary $N \in \mathbb{N}$. Since the proof is quite similar to Theorem 3.3, we omit the details.

Proposition 3.5. Let $Y$ be a Banach space. The pair $\left(\ell_{1}^{N} ; Y\right)$ has the $\mathbf{L}_{p, p}$ for operators if and only if given $\varepsilon>0$, a nonempty set $A \subset\{1, \ldots, N\}$ and $\left(\alpha_{j}\right)_{j \in A}$ with $\alpha_{j}>0$ for all $j \in A$ such that $\sum_{j \in A} \alpha_{j}=1$, there is $\eta=\eta\left(\varepsilon,\left(\alpha_{j}\right)_{j \in A}\right)>0$ such that whenever $\left(y_{j}\right)_{j \in A} \subset S_{Y}$ satisfies

$$
\left\|\sum_{j \in A} \alpha_{j} y_{j}\right\|>1-\eta,
$$

there are $z^{*} \in S_{Y^{*}}$ and $\left(z_{j}\right)_{j \in A} \subset S_{Y}$ such that

$$
z^{*}\left(z_{j}\right)=1 \quad \text { and } \quad\left\|z_{j}-y_{j}\right\|<\varepsilon
$$

for all $j \in A$.

It turns out that the AHSP (see Definition 3.1) implies the characterization of Proposition 3.5, as we show in the following theorem. This provide new examples of spaces $Y$ such that $\left(\ell_{1}^{N} ; Y\right)$ has the $\mathbf{L}_{p, p}$ for linear operators. In particular, if $Y$ is a uniformly convex Banach space, then the pair $\left(\ell_{1}^{N} ; Y\right)$ has the $\mathbf{L}_{p, p}$, a result that was already proved in [21, Proposition 2.10].

Theorem 3.6. Let $Y$ be a Banach space and $N \in \mathbb{N}$. If $Y$ has AHSP, then $\left(\ell_{1}^{N} ; Y\right)$ has the $\mathbf{L}_{p, p}$.

Proof. Assume that $Y$ has AHSP with a function $\eta$ and fix $\varepsilon>0$, a nonempty $A \subset\{1, \ldots, N\}$ and a sequence of positive numbers $\left(\alpha_{j}\right)_{j \in A}$ with $\sum_{j \in A} \alpha_{j}=1$. Take $0<\lambda<\min \left\{\varepsilon, \min \left\{\alpha_{j}: j \in A\right\}\right\}$ and assume that a sequence of vectors $\left(y_{j}\right)_{j \in A} \subset S_{Y}$ satisfies

$$
\left\|\sum_{j \in A} \alpha_{j} y_{j}\right\|>1-\eta(\lambda) .
$$

By the definition of AHSP, there are $B \subset A,\left\{z_{k}: k \in B\right\} \subset S_{X}$ and $z^{*} \in S_{Y^{*}}$ such that

$$
\sum_{k \in B} \alpha_{k}>1-\lambda, \quad\left\|z_{k}-x_{k}\right\|<\lambda, \quad \text { and } \quad x^{*}\left(z_{k}\right)=1
$$

for all $k \in B$. Since $\lambda<\min \left\{\alpha_{j}: j \in A\right\}, A=B$. By Proposition $3.5,\left(\ell_{1}^{N} ; Y\right)$ has the $\mathbf{L}_{p, p}$.

Corollary 3.7. Let $Y$ be a Banach space and $N \in \mathbb{N}$. If $Y$ is a

(a) finite dimensional space or

(b) uniformly convex space or

(c) lush space,

then the pair $\left(\ell_{1}^{N} ; Y\right)$ has the $\mathbf{L}_{p, p}$ for operators.

We also get a result about uniformly strongly exposed family. We say that a family $\left\{y_{\alpha}\right\}_{\alpha} \subset S_{Y}$ is uniformly strongly exposed with respect to a family $\left\{f_{\alpha}\right\}_{\alpha} \subset S_{Y^{*}}$, if there is a function $\varepsilon \in(0,1) \mapsto$ $\delta(\varepsilon)>0$ such that $f_{\alpha}\left(y_{\alpha}\right)=1$ for all $\alpha$ and $\operatorname{Re} f_{\alpha}(y)>1-\delta(\varepsilon)$ implies $\left\|y-y_{\alpha}\right\|<\varepsilon$ whenever $y \in B_{X}$.

Proposition 3.8. Let $Y$ be a Banach space and let $\left\{y_{\alpha}\right\}_{\alpha} \subset S_{Y}$ be a uniformly strongly exposed family with corresponding functionals $\left\{f_{\alpha}\right\}_{\alpha} \subset S_{Y^{*}}$. If $\left\{f_{\alpha}\right\}_{\alpha}$ is a norming subset for $Y$, then the pair $\left(\ell_{1}^{N} ; Y\right)$ has the $\mathbf{L}_{p, p}$.

Proof. Let $N \in \mathbb{N}$ and $A \subset\{1, \ldots, N\}$ be a nonempty finite subset. Let $\varepsilon>0$ and suppose there is $\alpha:=\left(\alpha_{j}\right)_{j \in A}$ such that $\alpha_{j}>0$ for all $j \in A$ and $\sum_{j \in A} \alpha_{j}=1$. Set $K_{\alpha}:=\min \left\{\alpha_{j}: j \in A\right\}$ and define

$$
\eta=\eta\left(\varepsilon,\left(\alpha_{j}\right)_{j \in A}\right):=K_{\alpha} \delta\left(\frac{\varepsilon}{2}\right)>0
$$


where $\varepsilon \mapsto \delta(\varepsilon)$ is the function for the family $\left\{y_{\alpha}\right\}_{\alpha}$. Let $\left(y_{j}\right)_{j \in A} \subset S_{Y}$ be such that

$$
\left\|\sum_{j \in A} \alpha_{j} y_{j}\right\|>1-\eta
$$

Since $\left\{f_{\alpha}\right\}$ is norming for $Y$, we may take $\alpha_{0}$ to be such that

$$
\sum_{j \in A} \alpha_{j} \operatorname{Re} f_{\alpha_{0}}\left(y_{j}\right)=\operatorname{Re} f_{\alpha_{0}}\left(\sum_{j \in A} \alpha_{j} y_{j}\right)>1-\eta .
$$

Then, for each $i \in A$, we have

$$
1-K_{\alpha} \delta\left(\frac{\varepsilon}{2}\right)<\sum_{j \in A} \alpha_{j} \operatorname{Re} f_{\alpha_{0}}\left(y_{j}\right) \leqslant \sum_{j \in A \backslash\{i\}} \alpha_{j}+\alpha_{i} \operatorname{Re} f_{\alpha_{0}}\left(y_{i}\right)=1-\alpha_{1}+\alpha_{i} \operatorname{Re} f_{\alpha_{0}}\left(y_{i}\right) .
$$

Therefore, for each $i \in A$, we have

$$
1-\operatorname{Re} f_{\alpha_{0}}\left(y_{i}\right)<\frac{K_{\alpha}}{\alpha_{i}} \delta\left(\frac{\varepsilon}{2}\right) \leqslant \delta\left(\frac{\varepsilon}{2}\right)
$$

which implies that $\operatorname{Re} f_{\alpha_{0}}\left(y_{i}\right)>1-\left(\frac{\varepsilon}{2}\right)$ for every $i \in A$. So, we have that $\left\|y_{i}-y_{\alpha_{0}}\right\|<\frac{\varepsilon}{2}$ for all $i \in A$. Thus, for every $n, m \in A$, we have that

$$
\left\|y_{n}-y_{m}\right\| \leqslant\left\|y_{n}-y_{\alpha_{0}}\right\|+\left\|y_{\alpha_{0}}-y_{m}\right\|<\varepsilon .
$$

Since $A \neq \emptyset$, choose $n_{0} \in A$ and set $z_{j}:=y_{n_{0}}$ for all $j \in A$. Then, $z_{j} \in S_{Y}$ and $\left\|z_{j}-y_{j}\right\|=\left\|y_{n_{0}}-y_{j}\right\|<\varepsilon$ for all $j \in A$. Finally, take $y^{*} \in S_{Y^{*}}$ to satisfy $y^{*}\left(z_{j}\right)=y^{*}\left(y_{n_{0}}\right)=1$. By Proposition $3.5,\left(\ell_{1}^{N} ; Y\right)$ has the $\mathbf{L}_{p, p}$.

\section{REFERENCES}

[1] M. D. Acosta, Denseness of norm attaining mappings, Rev. R. Acad. Cien. Serie A. Mat. 100 (2006), 9-30.

[2] M. D. Acosta, F. J. Aguirre and R. PayÁ, There is no bilinear Bishop-Phelps theorem, Israel J. Math. 93 (1996), $221-227$.

[3] M. D. Acosta, R. M. Aron, D. García And M. Maestre, The Bishop-Phelps-Bollobás theorem for operators, J. Funct. Anal. 294 (2008), 2780-2899.

[4] M. D. Acosta, J. Becerra-Guerrero, D. García and M. Maestre The Bishop-Phelps-Bollobás Theorem for bilinear forms, Trans. Amer. Math. Soc. 365 (2013), 5911-5932

[5] J. Alaminos, Y. S. Choi, S. G. Kim And R. PAYÁ, Norm attaining bilinear forms on spaces of continuous functions, Glasgow Math. J. 40 (1998), 359-365.

[6] A. Arias and J.D. FARmer, On the structure of tensor products of $\ell_{p}$-spaces, Pac. J. Math. 175(1) (1996), $13-37$.

[7] R. M. Aron, Y. S. Choi, S. K. Kim, H. J. Lee and M. Martín, The Bishop-Phelps-Bollobás version of Lindenstrauss properties A and B, Trans. Amer. Math. Soc. 367 (2015), 6085-6101.

[8] R. M. Aron, C. Finet And E. Werner, Some remarks on norm-attaining n-linear forms, in: Function spaces (Edwardsville, IL, 1994), 19-28, Lecture Notes in Pure and Appl. Math., 172, Dekker, New York, 1995.

[9] E. Bishop And R.R. Phelps, A proof that every Banach space is subreflexive, Bull. Amer. Math. Soc. 67 (1961) 97-98

[10] B. Bollobás, An extension to the theorem of Bishop and Phelps, Bull. London. Math. Soc. 2 (1970), 181-182.

[11] J.M. Borwein And J. VANDERWERFF, Dual Kadec-Klee norms and the relationships between Wijsman, slice and Mosco convergence, Michigan Math. J. 41(2) (1994), 371-387.

[12] J. Bourgain, On dentability and the Bishop-Phelps property, Israel J. Math. 28 (1977), $265-271$.

[13] Y. S. Chor, Norm attaining bilinear forms on $L^{1}[0,1]$, J. Math. Anal. Appl. 211 (1997), 295-300.

[14] Y.S. Choi And H.G. Song., The Bishop-Phelps-Bollobás theorem fails for bilinear forms on $\ell_{1} \times \ell_{1}, J$. Math. Anal. Appl. 360 2009, 752-7523.

[15] Y.S. Choi And S.K. Kim, The Bishop-Phelps-Bollobás property and lush spaces. J. Math. Anal. Appl. 390 (2012), no. $2,549-555$

[16] S. Dantas, Some kind of Bishop-Phelps-Bollobás property, Math. Nachr. 290 (2017), no. 5-6, 774-784.

[17] S. Dantas, D. García, S. K. Kim, H. J. Lee and M. Maestre, On the Bishop-Phelps-Bollobás theorem for multilinear mappings. Linear Algebra Appl. 532 (2017), 406-431

[18] S. Dantas, S. K. Kim and H. J. Lee, The Bishop-Phelps-Bollobás point property, J. Math. Anal. Appl. 444 (2016), 1739-1751

[19] S. Dantas, V. Kadets, S. K. Kim, H. J. Lee And M. Martín, On the pointwise Bishop-Phelps-Bollobás property for operators, Canadian J. Math. (to appear). https://arxiv.org/abs/1709.00032 
[20] S. Dantas, V. Kadets, S. K. Kim, H. J. Lee and M. Martín, There is no operatorwise version of the BishopPhelps-Bollobás property, Linear Mult. Algebra (to appear), https://arxiv.org/abs/1810.00684

[21] S. Dantas, S.K. Kim, H.J. Lee and M. Mazzitelli, Local Bishop-Phelps-Bollobás properties, J. Math. Anal. Appl. 468 (2018), no. 1, 304-323

[22] S.J. Dilworth and D. Kutzarova, Kadec-Klee properties for $\mathcal{L}\left(\ell_{p}, \ell_{q}\right)$, Function spaces (Edwardsville, IL, 1994), Lecture Notes in Pure and Appl. Math., 172, Dekker, New York, 1995, 71-83.

[23] C. Franchetti And R. PayÁ, Banach spaces with strongly subdifferentiable norm, Boll. Uni. Mat. Ital. VII-B, 1993, 45-70.

[24] C. Finet and R. PayÁ, Norm attaining operators from $L_{1}$ into $L_{\infty}$, Israel J. Math. 108 (1998), 139-143

[25] M. Fabian, P. Habala, P. Hàjek, V. M. Santalucía, J. Pelant and V. Zizler, Functional Analysis and InfiniteDimensional Geometry, Springer, 2000.

[26] G. Godefroy, V. Montesinos And V. Zizler, Strong subdifferentiability of norms and geometry of Banach spaces. Comment. Math. Univ. Carolin. 36 (1995), no. 3, 493502

[27] P. Hájek and J. Talponen, Note on Kadets Klee property and Asplund spaces, Proc. Amer. Math. Soc. 142 (2014), 3933-3939.

[28] M. I. KADEC, Spaces isomorphic to locally uniformly rotund spaces, Izv. Vyss. Uc. Zav. Matem. 1 (1959), $51-57$ and 1 (1961), 186-187.

[29] S. K. Kim And H. J. LeE, Uniform convexity and the Bishop-Phelps-Bollobás property, Canad. J. Math. 66, (2014), 373-386.

[30] S. K. Kim, H. J. LeE, and M. Martín, Bishop-Phelps-Bollobás property for bilinear forms on spaces of continuous functions. Math. Z. 283 (2016), no. 1-2, 157-167.

[31] J Lindenstrauss, On operators which attain their norm. Isr. J. Math. 1 139-148.

[32] R. A. RYAN, Introduction to tensor products of Banach spaces, Springer Monographs in Mathematics. Springer Verlag, London, 2002.

[33] D. SAIn, Smooth points in operator spaces and some Bishop-Phelps-Bollobás type theorems in Banach spaces, https://arxiv.org/abs/1802.07527v1

[34] J. TALPOnEn, Note on a kind of Bishop-Phelps-Bollobás property for operators, https://arxiv.org/abs/1707.03251

(Dantas) Department of Mathematics, Faculty of Electrical Engineering, Czech Technical University in Prague, Technická 2, 16627 Prague 6, Czech Republic

ORCID: 0000-0001-8117-3760

E-mail address: gildashe@fel.cvut.cz

(Kim) Department of Mathematics, Chungbuk National University, 1 Chungdae-Ro, Seowon-Gu, Cheonguu, Chungbuk 28644, Republic of Korea

ORCID: 0000-0002-9402-2002

E-mail address: skk@chungbuk.ac.kr

(Lee) Department of Mathematics Education, Dongguk University - Seoul, 04620 (Seoul), Republic of Korea ORCID: 0000-0001-9523-2987

E-mail address: hanjulee@dongguk.edu

(Mazzitelli) Universidad Nacional del Comahue, COniCET, Departamento de Matemática, Facultad de Economía y Administración, Neuquén, Argentina.

E-mail address: martin.mazzitelli@crub.uncoma.edu.ar 